\title{
Thermally Triggered Injectable Underwater Adhesives
}

Mehdi Vahdati, Guylaine Ducouret, Costantino Creton*, Dominique Hourdet*

Dr. M Vahdati, Dr. G Ducouret, Prof. Dr. C Creton, Prof. Dr. D Hourdet

Soft Matter Sciences and Engineering, ESPCI Paris, PSL University, Sorbonne University, CNRS, F-75005 Paris, France

Email: costantino.creton@espci.fr, dominique.hourdet@espci.fr

Key Words: thermoresponsive hydrogels, hydrophobic interactions, generic adhesion, underwater adhesion, Pressure-Sensitive Adhesives (PSAs)

\begin{abstract}
We report a novel bioinspired underwater adhesive based on the injectable aqueous solution of a graft copolymer with a thermoresponsive backbone, which turns into a sticky hydrogel just below body temperature. With this topology, the collapse of the backbones upon the thermal transition leads to the formation of a percolating network of strong hydrophobic domains. Similar to Pressure-Sensitive Adhesives (PSAs), the hydrogel goes through fibrillation and extensive energy dissipation in large deformations, giving it an edge over conventional chemical hydrogels which are typically elastic and inherently non-sticky. This capability comes from the hydrophobic nano-scaffold which resists large deformations to minimize its contact with water. Since hydrophobic interactions are not weakened in water, the behavior of the hydrogel is maintained in aqueous medium. Chemistry-insensitive adhesion of this hydrogel offers a major advantage over current injectable adhesives which rely on in-situ chemical crosslinking reactions with tissues.
\end{abstract}




\section{Main Text}

The growing demand for minimally-invasive alternatives to sutures and staples has been a major drive for research on tissue adhesives. ${ }^{[1-3]}$ A popular option has been to inject a fluid precursor and perform a covalent crosslinking in-situ (on the tissue), raising concerns about the toxicity of the reaction and the removability of the adhesive. In addition, the performance of these adhesives eventually relies on the chemistry of the tissue, which may be quite dynamic and different from one tissue to another. ${ }^{[2,4,5]}$

Marine organisms exploit a variety of interactions over different length and time scales to achieve resilient adhesion in their harsh environments. ${ }^{[6-9]}$ Numerous research works have drawn inspirations from these animals in developing underwater adhesives based on catechol chemistry, which contributes to interfacial interactions and bulk dissipation for underwater adhesion under certain conditions. ${ }^{[5,7,10,11]}$ More recent findings in this field highlight the significance of weaker molecular interactions such as cation- $\pi$ and hydrophobic interactions. ${ }^{[12-}$ ${ }^{15]}$ For example, the interfacial performance of DOPA in mussel adhesion depends on the hydrophobicity of adjacent amino acid residues. ${ }^{[13]}$

Yet, many bioinspired formulations still rely on specific interactions and crosslinking, for instance, using periodate which is toxic. Developing soft generic adhesives which can stick onto different surfaces in the presence of water, without swelling too much, remains a major challenge in the field. Such generic adhesives could be inspired from hydrophobic pressuresensitive adhesives (PSAs) that rely on a fine balance of viscoelasticity to make rapid and intimate contact with substrates and dissipate large amounts of energy upon detachment. This type of adhesion is mainly controlled by fine-tuning the viscoelastic properties of the PSA rather than by specific molecular interactions. ${ }^{[16,17]}$

Along those lines, several recent reports focused on the enhancement of underwater adhesion of complex coacervates by introducing short poly( $N$-isopropylacrylamide) (PNIPAM( side chains on polyelectrolyte backbones. ${ }^{[18-20]}$ These materials are injectable at 
room temperature, but form sticky hydrogels upon immersion in an aqueous medium above the transition temperature (also called Lower Critical Solution Temperature or LCST) of PNIPAM owing to both electrostatic and hydrophobic interactions. Meanwhile, Guo and coworkers also reported the reversible formation of stable hydrogels upon heating aqueous solutions of graft copolymers designed either with a thermoresponsive PNIPAM backbone and hydrophilic poly(N,N-dimethylacrylamide) (PDMA) side chains or with the opposite topology, i.e. PDMA backbone with PNIPAM side-chains. ${ }^{[21]}$ Although these two opposite topologies, with similar composition (50/50 in weight), display very similar linear viscoelastic properties in semi-dilute conditions, an extensive study performed on similar covalently cross-linked architectures has clearly highlighted the higher toughness of the gels when PNIPAM is in the backbone. ${ }^{[2,23]}$ In this case, the formation of a bicontinuous nanostructure with a highly concentrated percolating PNIPAM phase across the swollen PDMA matrix results in a very high level of mechanical reinforcement. On the other hand, in graft copolymers with the opposite topology, the short PNIPAM side chains can more easily dissociate from the micellar domains in large strain.

These previous studies form the basis of the current work investigating the use of responsive hydrophobic interactions in the design of a viscoelastic adhesive hydrogel. In order to target both injectability and switchability to a viscoelastic gel state, our design takes advantage of the original topology involving thermo-responsive hydrophobic interactions between macromolecular backbones stabilized with hydrophilic side chains. We synthesized a graft copolymer with a long PNIPAM backbone $\left(\mathrm{M}_{\mathrm{n}} \sim 440 \mathrm{~kg} \cdot \mathrm{mol}^{-1}\right)$ bearing short $\left(\sim 14 \mathrm{~kg} \cdot \mathrm{mol}^{-}\right.$ ${ }^{1}$ ) hydrophilic PDMA side chains (50-50 wt\%) via a grafting through procedure (more details in the SI and Figure 1S). At 8 wt\%, well into the entangled regime, the aqueous solution of this polymer turns into a stable hydrogel just below body temperature due to the thermal and structural transition of PNIPAM.

Figure 1A shows the evolution of the transition enthalpy of PNIPAM in an $8 \mathrm{wt} \%$ aqueous solution of PNIPAM-g-PDMA. The thermal transition of the backbone is sharp, with 
$\sim 90 \%$ of the final enthalpy obtained between $\sim 34$ and $40{ }^{\circ} \mathrm{C}$. At higher temperatures, hydrophobic interactions become stronger and the transition reaches its final stage by $45^{\circ} \mathrm{C}$. Given the high molecular weight of the backbone $\left(\mathrm{M}_{\mathrm{n}}\right.$, backbone $\left.=440 \mathrm{~kg} \cdot \mathrm{mol}^{-1}\right)$ and its graft architecture, a sharp transition similar to that of a PNIPAM homopolymer ( 4-6 kJ.mol ${ }^{-1}$ NIPAM $)$ was expected. ${ }^{[24,25]}$ Figure 1B probes this transition in terms of the thermothickening behavior (for the linear viscoelastic regime, see Figure 2S). Above the LCST, the complex viscosity rises by 2 orders of magnitude, mainly up to $40{ }^{\circ} \mathrm{C}$ followed by a pseudo-plateau. This change in properties parallels the increase in $G^{\prime}(T)$ with a crossover of the dynamic moduli $\left(G^{\prime}=G^{\prime \prime}\right)$ at $\sim 33{ }^{\circ} \mathrm{C}$ (Figure 3S) where the clear solution turns into a white, macroscopic gel (images in Figure 1C). As schematically proposed in Figure 1C, the only possible mechanism of gelation and the initial jump in the complex viscosity is the collapse of PNIPAM backbones into percolating hydrophobic domains across the swollen hydrophilic matrix. The domains then become more concentrated with temperature, leading to a gradual increase in complex viscosity.

Small Angle Neutron Scattering (SANS) measurements (Figure 4S) support the thermal transition and show a dramatic increase in scattering intensity at low q along with the formation of a two-phases morphology with sharp boundaries between hydrophobic and hydrophilic domains upon crossing the LCST $\left(q^{-4}\right.$ dependence in the intermediate regime). A more quantitative analysis of the scattering pattern at $50{ }^{\circ} \mathrm{C}$ shows that the average distance between PNIPAM domains (red phase in Figure 1C) is $\geq 750 \AA$, while their concentration is about 75 $\mathrm{wt} \%$, in good agreement with the literature. ${ }^{[21,26]}$

The adhesive properties of hydrogel layers $(400 \mu \mathrm{m})$ at different temperatures below and above gelation were tested following a probe tack procedure specifically adapted to thermoresponsive adhesives (Figure 5S and Figure 6S). We worked with standard stainless steel plates as a simple model system to avoid measuring additional contributions to adhesion energy from dissipation in the soft substrate and/or the penetration of the adhesive into the substrate. 
In air, highly confined layers of $8 \mathrm{wt} \%$ PNIPAM-g-PDMA equilibrated (10 min) at each temperature were debonded at a nominal strain rate $(\dot{\varepsilon})$ of $2.5 \mathrm{~s}^{-1}$. The nominal stress-nominal strain curves are plotted in Figure 2A. Just below the transition temperature, i.e. at $32{ }^{\circ} \mathrm{C}$, the material is a viscous fluid ( $\eta^{*} \sim 30$ Pa.s) forming a single thinning filament in the center of the probe (Figure 2B) following the behavior expected for the confined layer between two parallel plates: a quick drop of stress to zero (similar to the prediction for a Newtonian fluid, $\sigma_{N} \propto h^{-5}$ where $\mathrm{h}$ is the probe position) following a small initial peak marking the penetration of air fingers pushing the liquid layer inwards. ${ }^{[27,28]}$

As soon as the sol-gel temperature is crossed, the adhesive behavior of the hydrogel (Figure 2A) bears qualitative, but striking resemblance to that of PSAs: 1) a sharp increase in the nominal stress during homogenous deformation of the layer, 2$)$ a marked peak $\left(\sigma_{\max }\right)$ due to the occurrence and growth of different instabilities in the bulk of the layer (which will be discussed shortly), 3) the drop of the stress to a plateau which coincides with the onset of fibrillation, 4) a second growth of stress as the filaments are stretched to very large strains (Figure 2B); a hallmark of strain-hardening, 5) a cohesive failure at $\varepsilon_{\max } \cdot{ }^{[17]}$ The stress-strain curves evolve with temperature, limiting the maximum strains at detachment while increasing stress levels as the hydrogel becomes more cohesive. A qualitatively similar trend of limited fibrillation is well established for acrylate-based PSAs when decreasing the average molecular weight between crosslinks. ${ }^{[29,30]}$

The stress-strain curves may give the impression that the filaments go through more strain-hardening at higher temperatures. In order to be able to compare the hardening, it is more appropriate to consider the relative enhancement in stress at each temperature. We thus introduce a "hardening ratio" defined as the ratio between the second peak in stress and the minimum stress measured after the initial drop. This ratio is indirectly related to the nonlinear relaxation response of the hydrogel, which is accelerated in large deformations but is technically difficult to measure directly, for instance, using extensional rheology. 
The fact that the hardening ratio remains unchanged around 2.0 (Figure 2C) is consistent with the molecular picture proposed in Figure 1C. At equilibrium (rest), the hydrophobicity of the load-bearing network of PNIPAM seeks to minimize its contact with water molecules. Therefore, stretching this structure out of equilibrium, exposing the hydrophobic domains to water, is energetically unfavorable. As these domains become more hydrophobic and more concentrated in polymer (and thus mechanically stronger) with increasing temperature, the hydrogel becomes more cohesive and the stress levels rise but the molecular picture must remain the same as shown by the self-similar shape of the tack curves above the LCST as well as that of the scattering intensity in SANS (Figure 4S). We have also observed this marked hardening behavior in shear (see Figure 7S).

Figure 2D summarizes the above trends into a single parameter, $\mathrm{W}_{\mathrm{adh}}$, or the energy required to detach a unit surface area of the adhesive $\left(\mathrm{W}_{\mathrm{adh}}\right.$ is proportional to the area under the curve for a given initial thickness). At a nominal strain rate of $2.5 \mathrm{~s}^{-1}$, reasonably close to real applications, the material is in a fluid state at $32{ }^{\circ} \mathrm{C}$ with a negligible $\mathrm{W}_{\text {adh }}\left(0.3 \mathrm{~J} \cdot \mathrm{m}^{-2}\right)$. Above the thermal transition, the low viscosity fluid immediately becomes a viscoelastic, sticky gel. At physiological temperature, the hydrogel already requires more than $20 \mathrm{~J} \cdot \mathrm{m}^{-2}$ for detachment, while the $\mathrm{W}_{\text {adh }}$ at $50{ }^{\circ} \mathrm{C}$ comes close to commercial removable labels such as Post-It ${ }^{\circledR}$ notes.

Despite the general similarity of the temperature-induced increase in adhesion energy with the enthalpy of the transition and the complex viscosity (Figure 1A and B), the increase in adhesion energy is much larger and sharper with $\mathrm{T}$. We believe that the breakage and reformation of the hydrogen bonds involved in the thermal transition of PNIPAM do not occur all at once; instead, it is a gradual process which is difficult to track in the very beginning. Likewise, the formation of the percolating network does not cause a dramatic change in linear mechanical properties. However, the formation of a percolating structure changes the nonlinear response dramatically so that stretching numerous stable fibrils (as opposed to a single fibril in the fluid state) to large deformations dissipates considerably more energy. 
Adhesiveness of generic adhesives (PSAs) is primarily due to their soft, viscoelastic nature rather than chemistry-specific reactions (with substrates or within the adhesive). They are capable of making intimate contact with substrates owing to their fluid-like nature while dissipating tremendous amounts of energy before failure. Upon detachment, they go through extensive fibrillation because of their lightly crosslinked and highly-branched molecular architecture which gives them just enough elasticity to induce strain-hardening in the filaments. ${ }^{[17,31]}$ Given the similarity of PNIPAM-g-PDMA hydrogels to soft PSAs, the dissipative capability of the hydrogel at different nominal strain rates was studied at $50{ }^{\circ} \mathrm{C}$ (Figure 3).

The marked strain-rate dependence of stress-strain curves in Figure 3A is a manifestation of an increase in both stiffness and viscoelastic dissipation. At small strain, the measured $\sigma_{\max }$ increases with strain rate due to the soft, viscoelastic nature of the hydrogels, which requires higher stresses to cause bulk instabilities. ${ }^{[31,32]}$ At larger deformations $(\varepsilon>2)$, no strainhardening (hardening ratio of 1.0, Figure 3B) was observed at small strain rates $\left(\dot{\varepsilon}<0.25 \mathrm{~s}^{-1}\right)$ and the layers could not be stretched beyond $\varepsilon_{\max } \approx 3-4$. In this case, the material behaved like a weak gel with adhesive failure before extensive deformation. At $\dot{\varepsilon} \geq 0.25 \mathrm{~s}^{-1}$, strain-hardening appeared and further increased with strain rate allowing larger stresses and strains to be achieved before cohesive failure. In fact, strain-hardening in the filaments enables them to maintain higher stress levels and consequently dissipate larger amounts of energy. These observations are consistent with the increase in both dynamic moduli as a function of frequency (see Figure 8S). Similar to PSAs, this is reflected in the power-law increase of $\mathrm{W}_{\text {adh }}$ with nominal strain rate $\left(\mathrm{W}_{\mathrm{adh}} \propto \dot{\varepsilon}^{0.34}\right.$, shown by the fit in Figure $\left.3 \mathrm{~B}\right) .^{[31]}$

Both the hardening ratio and $\mathrm{W}_{\mathrm{adh}}$ suggest two regimes of strain rate-dependence below and above $0.2 \mathrm{~s}^{-1}$. In order to clarify the failure mechanisms in each regime, Figure 3C shows the debonded layers immediately after detachment. In the low strain rates regime, undulating finger-like protrusions appear mainly at the periphery, but also in the bulk, of the layer close to 
$\sigma_{\text {max }}$. The fingers, known as the Saffman-Taylor instabilities, ${ }^{[33]}$ are caused by the intrusion of air (low viscosity medium) into the highly confined layer as it is initially sucked inwards towards the center of the probe under tension. ${ }^{[34,35]}$ Similar instabilities have been extensively reported for confined layers of soft, (visco)elastic solids as well as fluids. ${ }^{[27,28,32,36-38]}$ Along with the stress strain curves, these pictures reveal that a higher strain rate induces more fingering instabilities but they only affect a small area of the layer and do not result in much fibrillation.

At higher strain rates, the fingers appear to have smaller wavelengths and extend shorter distances into the bulk of the layer. Meanwhile, the center of the probe appears poked with other instabilities which resemble cavities grown out of preexisting defects such as trapped air bubbles. The general requirement for the occurrence of cavitation in highly confined layers of incompressible, soft, elastic solids is that the hydrostatic pressure component of the tensile load should exceed atmospheric pressure $(100 \mathrm{kPa}) .{ }^{[17,37]}$ Looking back at Figure $3 \mathrm{~A}$, the stress levels are arguably low for cavitation but the hydrostatic pressure component of the tensile load under the probe remains parabolic before the occurrence of instabilities $\left(\sigma_{\text {true }, r=0}=2 \times\right.$ $\left.\sigma_{\max }\right){ }^{[37,39]}$ Given that the average (nominal) stress stands at $50 \mathrm{kPa}$ at $0.25 \mathrm{~s}^{-1}$, the hydrogel is possibly at the threshold for cavitation.

In fact, Figure 3C provides further evidence for the viscoelastic behavior of the hydrogel in large deformations. In liquid layers, cavitation preferentially occurs in the center of the probe (highest hydrostatic pressure) while in solid systems it usually takes place uniformly across the entire layer. ${ }^{[37,40]}$ In this case, the hydrogel is somewhere in between those two limits where bulk cavities occur under an increasingly larger fraction of the probe with strain rate $(\sim 30$ and $45 \%$ of the entire area at 0.25 and $2.5 \mathrm{~s}^{-1}$, respectively). This is due to the occurrence of fingering instabilities in the soft adhesive before the necessary tensile stress for full cavitation is reached. The side-view picture at $0.25 \mathrm{~s}^{-1}$ shows that the filaments formed at very large deformations grow out of the fingers and cavities. It thus follows that more extensive initial deformation of the bulk (into fingers and cavities) is a prerequisite for substantial fibrillation. 
The filaments formed must then be stable for large strains to be achieved. ${ }^{[17]}$ In effect, a larger number of stable filaments withstand a higher stress.

It may be argued that at small strains the hydrogels are elastic ( $\left.G^{\prime}>G^{\prime \prime}\right)$, but we should bear in mind that linear rheology is based on small deformations $(0.1$ to $10 \%)$ to maintain the microstructure of the hydrogel. Even then, the hydrogel features a frequency-dependent loss modulus of $\sim 0.1 \mathrm{kPa}$, meaning it retains some dissipative nature. Probe tack is performed in tensile mode on a highly-confined layer, thus applying a very complex stress field to the material in large deformations. Therefore, direct comparison of the two techniques without considering their inherent differences are misleading for structured materials.

The mechanical properties of the hydrogel in water were studied at high temperature in fully-immersed conditions. Underwater linear rheology was performed upon addition of preheated water after contact in air, where the evolution of the dynamic moduli were recorded over time (Figure 4A). As soon as the solution is surrounded with the hot aqueous medium, both moduli increase, with G' exceeding G”, almost instantaneously. Within $10 \mathrm{~min}$, the hydrogel is well into equilibrium with comparable moduli to those measured in air, and its volume and modulus remains stable for at least $15 \mathrm{hr}$. This indicates that there is no signature of excess swelling or shrinkage in this confined configuration, which would otherwise raise concerns regarding residual stresses and/or delamination from the probe.

Underwater probe tack experiments were performed following the same procedure, but 10 min after immersion in preheated water to ensure equilibrium (according to linear rheology). Figure 4B compares stress-strain curves obtained under the same conditions $\left(\dot{\varepsilon}=0.25 \mathrm{~s}^{-1}\right)$ in air and in water, along with the respective adhesion energies. As expected, the initial peak in stress remains almost intact since the contact was made in air and the dynamic moduli of the hydrogel are the same in both media. Like in air, stable filaments are formed and stretched to large deformations in water (Figure 4C) but the fibrillated structure is formed at a relatively lower stress. Additionally, the strain-hardening is largely suppressed in water, with the 
hardening ratio falling from 2 to 1.1 . Overall, the adhesive retains $60-70 \%$ of its $d r y$ performance $\left(\mathrm{W}_{\mathrm{adh}}\right)$ when tested under water regardless of the strain rate (Figure 9S) and time immersion (for an experiment after 15 h, see Figure 10S).

The images in Figure 4C further confirm that the hydrogel shows generic adhesion under water, where energy dissipated to create and stretch stable filaments manifests itself as adhesiveness. In this case, it is pockets of water, rather than air, which intrude the layer as fingering instabilities occur. The shape of the debonded layer reveals the occurrence of cavities in the bulk as well, which is consistent with the peak stress and the highly fibrillated layer in detachment. The debonded layer seems less extensively deformed compared to the same experiment in air (Figure 3C). One reason might be that Saffman-Taylor instabilities depend on the viscosity ratios of the layer and the intruding medium $\left(\eta_{\text {water }} \gg \eta_{\text {air }}\right)$. Another possible explanation is the swelling of the PDMA domains in the thin filaments formed. Indeed, the characteristic time for microgel swelling, assuming a radius of $100 \mu \mathrm{m}$ and a cooperative diffusion coefficient of $3.10^{-7} \mathrm{~cm}^{2} \cdot \mathrm{s}^{-1}$, is close to the duration of the tack experiments $(10-30$ S). ${ }^{[41,42]}$

This adhesive hydrogel is too soft for structural, industrial applications, but is already stronger than many similar adhesive hydrogels relying on weak physical interactions tested under experimentally comparable conditions. ${ }^{[18-20,43]}$ This claim does not concern bioinspired systems taking advantage of stronger, and in most cases, irreversible interactions such as those based on catechol chemistry. ${ }^{[44,45]}$ However, there are several instances of stronger underwater adhesives based on electrostatic interactions. ${ }^{[9,46,47]}$ Yet, the water content, which plays a determining role in the mechanical properties of these systems, should not be overlooked. For example, the coacervates prepared by Lawrence and Lapitsky, ${ }^{[46]}$ which featured higher bond strengths in lap shear, contain less than $40 \mathrm{wt} \%$ water as opposed to $92 \mathrm{wt} \%$ in the present work. Hydrophobic PSAs, on the other hand, lose up to $95 \%$ of their performance even in wet contact, 
with reported adhesion energies in the range of 2-26 J.m $\mathrm{m}^{-2}$ at a strain rate of $100 \mathrm{~s}^{-1}$ (compared to more than $40 \mathrm{~J} . \mathrm{m}^{-2}$ measured at $2.5 \mathrm{~s}^{-1}$ in our case). ${ }^{[44,48]}$

The important requirement for injectability is the transition from a viscous fluid to a viscoelastic gel. We have demonstrated that within this range of compositions and polymer architecture, this is quite achievable and the adhesive properties obtained are adequate for a temporary tissue adhesive. The advantage of this strategy is to provide a quick stick solution with a material free of potentially toxic reactive components (PNIPAM is not toxic, only the monomer is ${ }^{[49]}$ ) and to take advantage of viscoelasticity rather than specific interactions to stick. This physical hydrogel marks the first instance of using hydrophobic interactions to make a generic underwater adhesive in a similar way to standard PSA tapes.

\section{Supporting Information}

Supplementary Information, including the experimental section and complementary results, is provided. A video demonstrating the adhesion of two pieces of pork skin using this hydrogel at different temperatures is provided.

\section{Acknowledgements}

This project is part of the BioSmartTrainee Network. The project has received funding from the European Union's Horizon 2020 research and innovation programme under the Marie Sklodowska-Curie grant agreement no. 642861.

Received: ((will be filled in by the editorial staff))

Revised: ((will be filled in by the editorial staff)) Published online: ((will be filled in by the editorial staff))

\section{References}

[1] A. H. Hofman, I. A. van Hees, J. Yang, M. Kamperman, Adv. Mater. 2018, 30, 1704640.

[2] F. Scognamiglio, A. Travan, I. Rustighi, P. Tarchi, S. Palmisano, E. Marsich, M. Borgogna, I. Donati, N. De Manzini, S. Paoletti, J. Biomed. Mater. Res. - Part B Appl. Biomater. 2016, 104, 626.

[3] J. Yang, R. Bai, B. Chen, Z. Suo, Adv. Funct. Mater. 2019, 1901693, 1.

[4] C. Ghobril, M. W. Grinstaff, Chem. Soc. Rev. 2015, 44, 1820.

[5] A. P. Duarte, J. F. Coelho, J. C. Bordado, M. T. Cidade, M. H. Gil, Prog. Polym. Sci. 2012, 37, 1031.

[6] J. H. Waite, J. Exp. Biol. 2017, 220, 517.

[7] B. P. Lee, P. B. Messersmith, J. N. Israelachvili, J. H. Waite, Annu. Rev. Mater. Res. 2011, 41, 99.

[8] R. J. Stewart, T. C. Ransom, V. Hlady, J. Polym. Sci. Part B Polym. Phys. 2011, 49, 757. 
[9] H. Shao, R. J. Stewart, Adv. Mater. 2010, 22, 729.

[10] R. Pinnaratip, M. S. A. Bhuiyan, K. Meyers, R. M. Rajachar, B. P. Lee, Adv. Healthc. Mater. 2019, 1801568, 1.

[11] P. J. M. Bouten, M. Zonjee, J. Bender, S. T. K. Yauw, H. Van Goor, J. C. M. Van Hest, R. Hoogenboom, Prog. Polym. Sci. 2014, 39, 1375.

[12] E. Danner, J. N. Israelachvili, W. Wei, D. R. Miller, J. Yu, S. Das, Y. Kan, Y. Chen, M. Rapp, J. H. Waite, Proc. Natl. Acad. Sci. 2013, 110, 15680.

[13] J. Yu, J. H. Waite, J. N. Israelachvili, C. Broomell, W. Wei, J. Am. Chem. Soc. 2012, $135,377$.

[14] S. Kim, A. Faghihnejad, Y. Lee, Y. S. Jho, H. Zeng, D. S. Hwang, J. Mater. Chem. B 2015, 3, 738 .

[15] M. Valtiner, S. H. Donaldson, M. A. Gebbie, J. N. Israelachvili, J. Am. Chem. Soc. 2012, 134, 1746.

[16] C. Creton, MRS Bull. 2003, 434.

[17] C. Creton, M. Ciccotti, Reports Prog. Phys. 2016, 79, 46601.

[18] M. Dompé, F. J. Cedano-Serrano, O. Heckert, N. van den Heuvel, J. van der Gucht, Y. Tran, D. Hourdet, C. Creton, M. Kamperman, Adv. Mater. 2019, 1808179, 1808179.

[19] M. Dompé, F. J. Cedano-Serrano, M. Vahdati, L. van Westerveld, D. Hourdet, C. Creton, J. van der Gucht, T. Kodger, M. Kamperman, Adv. Mater. Interfaces 2019, 1901785, DOI 10.1002/admi.201901785.

[20] M. Dompé, F. J. Cedano-Serrano, M. Vahdati, U. Sidoli, O. Heckert, A. Synytska, D. Hourdet, C. Creton, J. van der Gucht, T. Kodger, M. Kamperman, Int. J. Mol. Sci. 2020, 21, DOI 10.3390/ijms21010100.

[21] H. Guo, A. Brûlet, P. R. Rajamohanan, A. Marcellan, N. Sanson, D. Hourdet, Polym. (United Kingdom) 2015, 60, 164.

[22] H. Guo, N. Sanson, D. Hourdet, A. Marcellan, Adv. Mater. 2016, $28,7043$.

[23] H. Guo, C. Mussault, A. Brûlet, A. Marcellan, D. Hourdet, N. Sanson, Macromolecules 2016, 49, 4295.

[24] H. G. Schild, D. A. Tirrell, J. Phys. Chem. 1990, 94, 4352.

[25] L. Petit, L. Bouteiller, A. Brûlet, F. Lafuma, D. Hourdet, Langmuir 2007, 23, 147.

[26] F. Afroze, E. Nies, H. Berghmans, J. Mol. Struct. 2000, 554, 55.

[27] D. Derks, A. Lindner, C. Creton, D. Bonn, J. Appl. Phys. 2003, 93, 1557.

[28] J. Nase, D. Derks, A. Lindner, Phys. Fluids 2011, 23, DOI 10.1063/1.3659140.

[29] A. Zosel, Int. J. Adhes. Adhes. 1998, 18, 265.

[30] M. M. Feldstein, V. G. Kulichikhin, S. V. Kotomin, T. A. Borodulina, M. B. Novikov, A. Roos, C. Creton, J. Appl. Polym. Sci. 2006, 100, 522.

[31] H. Lakrout, P. Sergot, C. Creton, J. Adhes. 1999, 69, 307.

[32] H. Lakrout, C. Creton, D. Ahn, K. R. Shull, Macromolecules 2001, 34, 7448.

[33] G. T. P. G. Saffman, Proc. R. Soc. A 1958, DOI https://doi.org/10.1098/rspa.1958.0085.

[34] J. S. Biggins, Z. Wei, L. Mahadevan, Epl 2015, 110, DOI 10.1209/02955075/110/34001.

[35] M. Ben Amar, D. Bonn, Phys. D Nonlinear Phenom. 2005, 209, 1.

[36] T. D. Dimitrova, D. Johannsmann, N. Willenbacher, A. Pfau, Langmuir 2003, 19, 5748.

[37] K. R. Shull, C. Creton, J. Polym. Sci. Part B Polym. Phys. 2004, 42, 4023.

[38] K. R. Shull, C. M. Flanigan, A. J. Crosby, Phys. Rev. Lett. 2000, 84, 3057.

[39] R. E. Webber, K. R. Shull, A. Roos, C. Creton, Phys. Rev. E - Stat. Physics, Plasmas, Fluids, Relat. Interdiscip. Top. 2003, 68, 11.

[40] A. Chiche, J. Dollhofer, C. Creton, Eur. Phys. J. E 2005, 17, 389.

[41] E. Sato Matsuo, T. Tanaka, J. Chem. Phys. 1988, 89, 1695. 
[42] Y. Li, T. Tanaka, J. Chem. Phys. 1990, 92, 1365.

[43] M. Guvendiren, P. B. Messersmith, K. R. Shull, Biomacromolecules 2008, 9, 122.

[44] S. K. Clancy, A. Sodano, D. J. Cunningham, S. S. Huang, P. J. Zalicki, S. Shin, B. K. Ahn, Biomacromolecules 2016, 17, 1869.

[45] H. Chung, P. Glass, J. M. Pothen, M. Sitti, N. R. Washburn, Biomacromolecules 2011, $12,342$.

[46] P. G. Lawrence, Y. Lapitsky, Langmuir 2015, 31, 1564.

[47] H. Shao, K. N. Bachus, R. J. Stewart, Macromol. Biosci. 2009, 9, 464.

[48] S. Poivet, P. Fabre, F. Nallet, K. Schierholz, G. Abraham, É. Papon, Y. Gnanou, R. Ober, O. Guerret, N. E. El-Bounia, Eur. Phys. J. E 2006, 20, 273.

[49] H. Vihola, A. Laukkanen, L. Valtola, H. Tenhu, J. Hirvonen, Biomaterials 2005, 26, 3055.
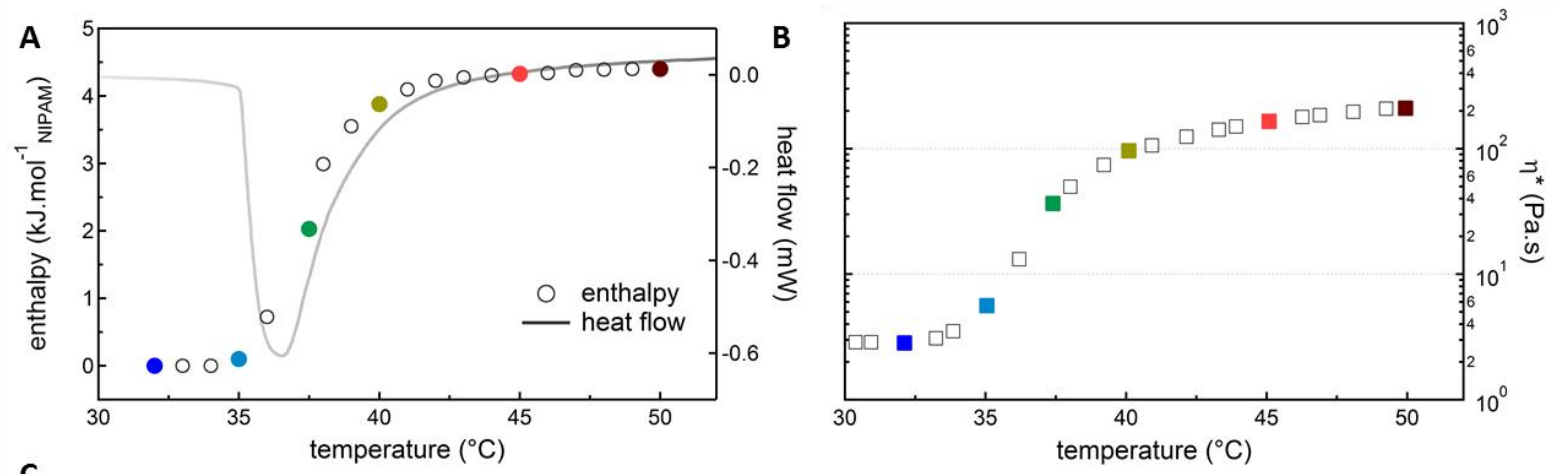

C
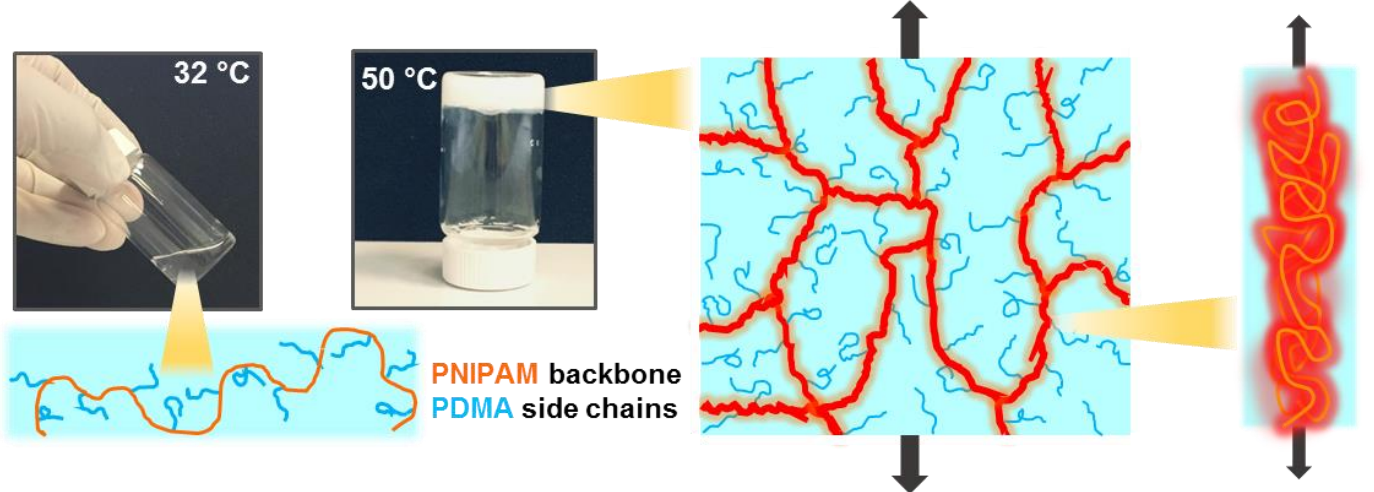

Figure 1. A. Heat flow and enthalpy of thermal transition of PNIPAM in 8 wt $\%$ PNIPAM-gPDMA at different temperatures heated at $2{ }^{\circ} \mathrm{C} \cdot \mathrm{min}^{-1}$. B. The corresponding evolution of the complex viscosity. C. Injectable $8 \mathrm{wt} \%$ solution and the sticky hydrogel formed at 32 and $50{ }^{\circ} \mathrm{C}$, respectively. The cartoons show the graft copolymer and the strong nanoscaffold made of collapsed, hydrophobic backbones, respectively. 

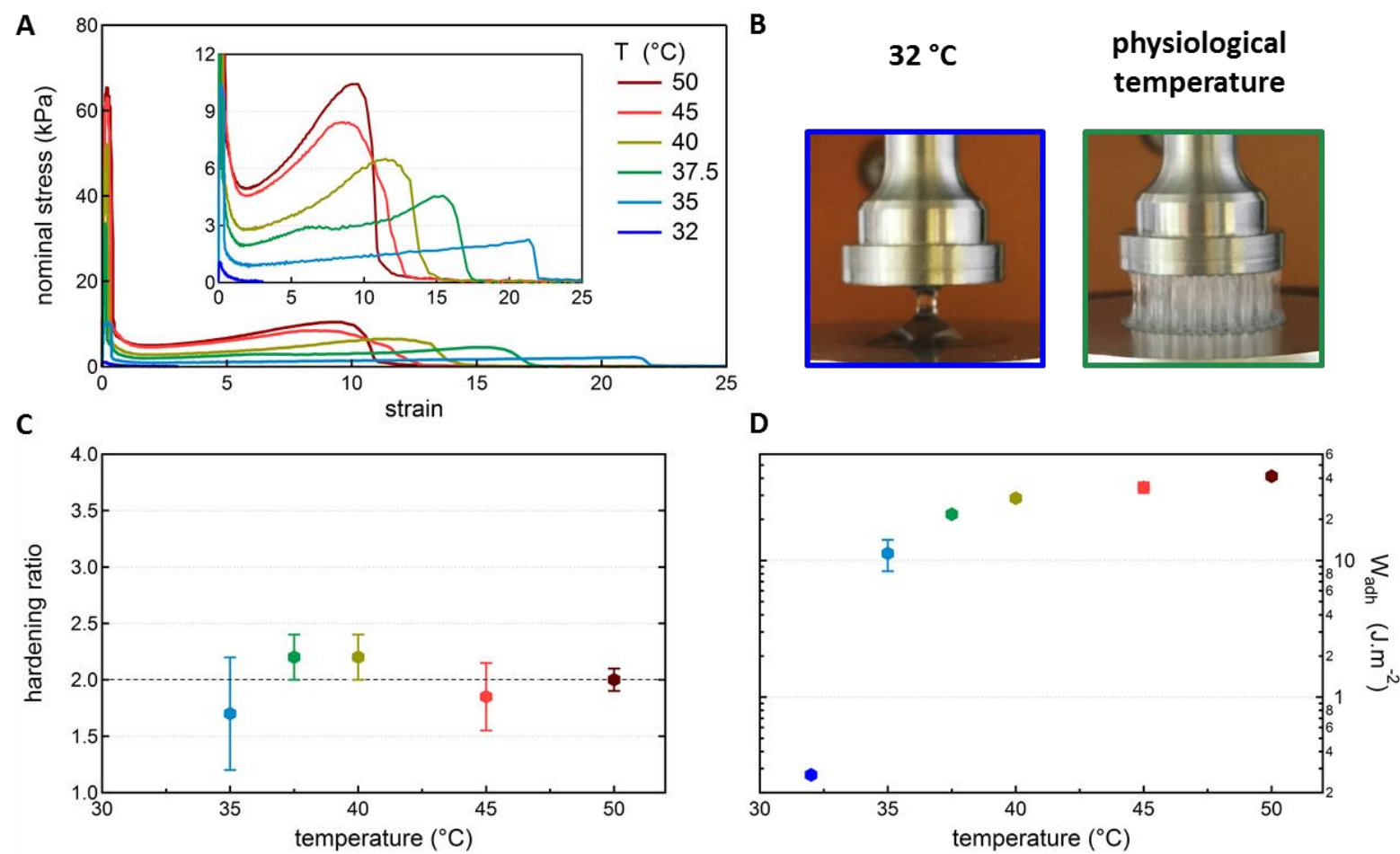

Figure 2. A. Nominal stress-strain plots from probe tack experiments after equilibration at different temperatures $\left(10 \mathrm{~min}, \mathrm{~h}_{0}=400 \mu \mathrm{m}, \dot{\varepsilon}=2.5 \mathrm{~s}^{-1}\right)$, with the plateau stress region magnified in the inset. B. Side-view images from experiments at 32 and $37.5^{\circ}$ C. C. and D. The corresponding hardening ratios and adhesion energies $\left(\mathrm{W}_{\mathrm{adh}}\right)$ at different temperatures. The color code is consistent among all the data on Figure 1 and Figure 2.
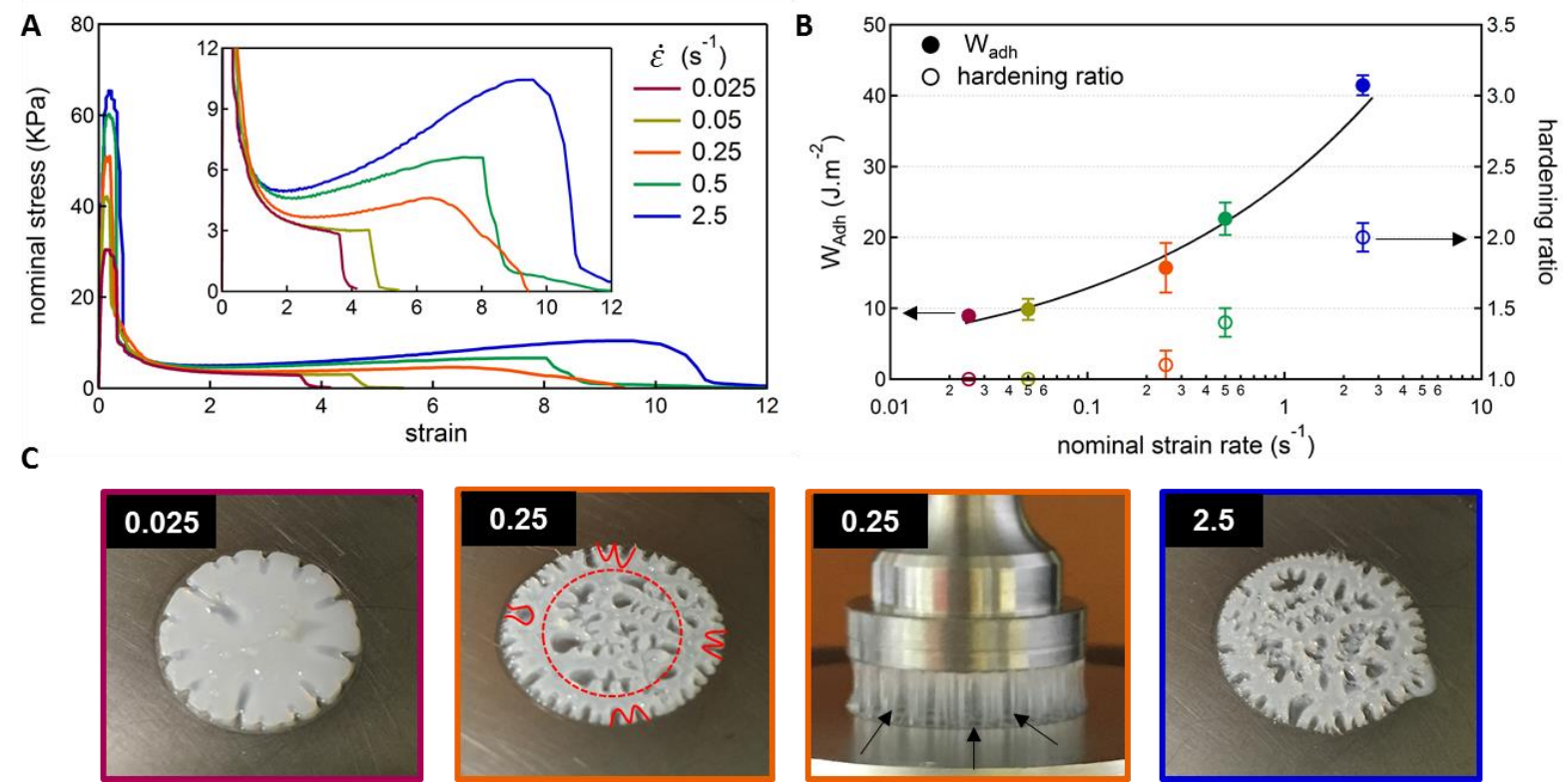

Figure 3. A. Nominal stress-strain curves from probe tack experiments on $8 \mathrm{wt} \%$ PNIPAM-gPDMA hydrogel at increasing nominal strain rates $\left(\mathrm{h}_{0}=400 \mu \mathrm{m}\right.$, at $\left.50{ }^{\circ} \mathrm{C}\right)$. The inset magnifies the stress plateau region. B. The corresponding adhesion energies $\left(\mathrm{W}_{\mathrm{adh}}\right)$ and hardening ratios. The black curve is the power-law fit. C. Images immediately after detachment from the probe. A side-view image where cavities are visible through the filaments is presented. 

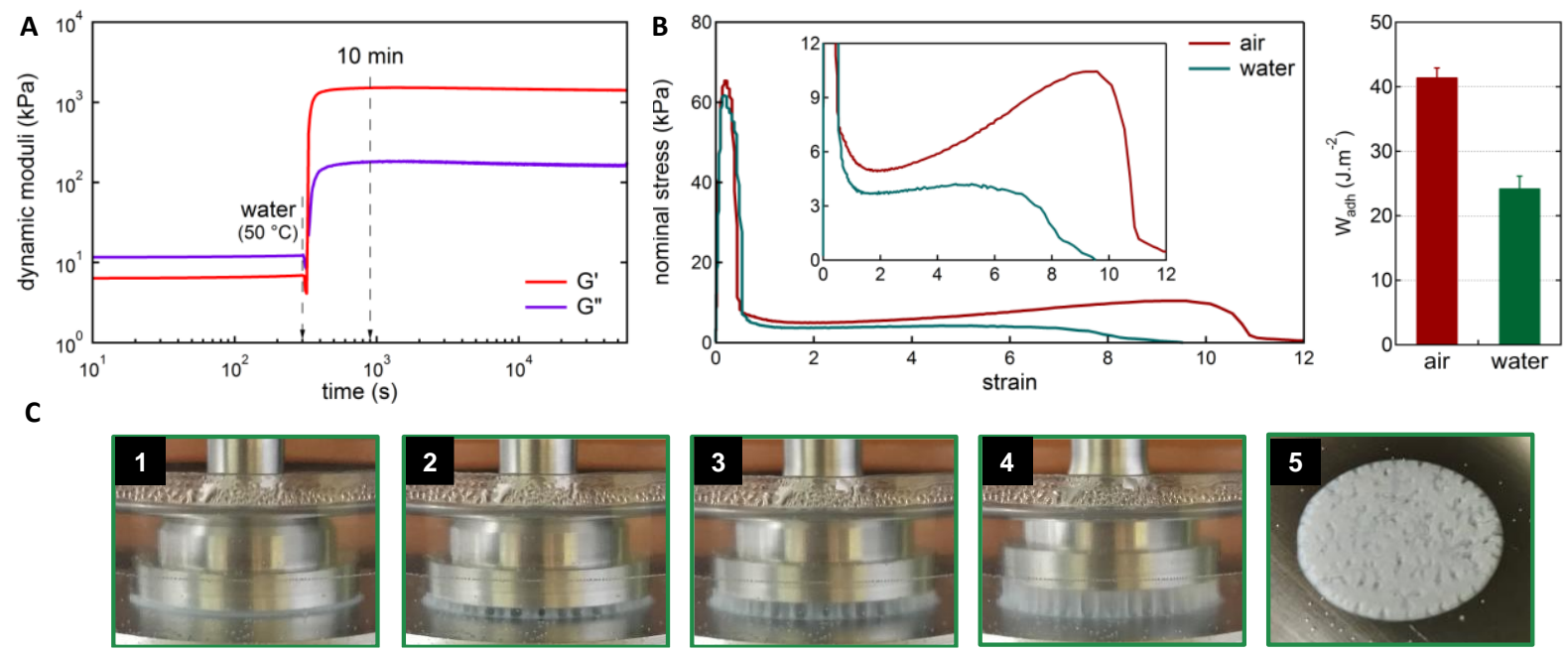

Figure 4. A. Temporal evolution of the dynamic moduli upon immersion in preheated water $\left(50{ }^{\circ} \mathrm{C}, 2 \mathrm{~Pa}, 1 \mathrm{~Hz}\right)$. B. Comparison of tack experiments performed in air and in preheated water at $50{ }^{\circ} \mathrm{C}\left(10 \mathrm{~min}, \mathrm{~h}_{0}=400 \mu \mathrm{m}, \dot{\varepsilon}=2.5 \mathrm{~s}^{-1}\right)$, and the corresponding adhesion energies. C. Sideview images from different stages of debonding in water. The last image was taken postdetachment. 